\title{
Editorial Perspective: Global Surgery: Measuring the Impact
}

\author{
K. A. Kelly McQueen
}

Published online: 30 August 2013

(c) Société Internationale de Chirurgie 2013

Surgery and safe anesthesia are important contributors to global public health. This recent recognition occurs as noncommunicable disease is growing and surpassing the role of communicable disease [1], including HIV/AIDS, in contributing to disability and premature death in lowincome countries (LICs.) Surgical intervention for trauma, cancer, obstetric emergencies, and other essential surgical conditions [2] is required to affect the global burden of disease (GBD). Prioritizing surgical interventions for the conditions in LICs contributing most greatly to the GBD and for which the surgery is cost-effective and proven [3] has created a focus on treating trauma, some cancers, cataracts, simple congenital defects, and obstetric emergencies. However, without safe anesthesia, even the most highly prioritized procedures may not have the intended impact if anesthesia outcomes are poor [4-6].

The provision of surgery and safe anesthesia is not the end point. The impact of surgery and verification of safe anesthesia must be evaluated through complication rates, follow-up, and outcome measures. These data are surprisingly difficult to collect in LICs. In a majority of LICs, patients are cared for in the hospital by family members, and there is little follow-up or documentation following procedures or treatments. A patient's condition after hospital discharge is rarely known in LICs, and complications are not often reported to the doctors providing services in these settings. Even death is most often unrecorded, as most patients die at home, and death certificates in LICs are not issued [7].

K. A. K. McQueen ( $₫)$

Department of Anesthesiology, Vanderbilt Institute for Global

Health, Vanderbilt University Medical Center, 1301 Medical

Center Drive, \#4648 TVC, Nashville, TN 37232, USA

e-mail: kamcqueen@gmail.com; kelly.mcqueen@vanderbilt.edu
Measuring the impact of surgical intervention and the outcomes related to surgery and anesthesia in LICs is a challenge. Surgical and anesthesia-related morality rates may reflect the current state of surgical care by country, and are generally recorded in LICs. With this in mind, there is consensus among surgeons and anesthesiologists committed to global health that perioperative death divided by total surgical interventions, the perioperative mortality rate (POMR), is an initial health indicator for surgical care. The POMR is a generally available, reportable data point that could serve as a starting point toward comparing surgical services at a population level [8].

Defining the POMR, defined as death occurring in the operating room or within the first $24 \mathrm{~h}$ (POMR 24) divided by total interventions performed is an endpoint of value, potentially similar to the maternal mortality rate (MMR). Since its introduction the MMR has transformed support for improving outcomes in the perinatal period. Like the MMR, the POMR is nonspecific and requires thoughtful application. It is important to note that the POMR is suggested as a surgical health indicator, which provides basic population level information and reveals no specific information for stratification or analysis. However, health indicators, especially mortality rates, are powerful summary statistics within global health, and following their trends has provided valuable feedback on interventions for improvement. Therefore recording the POMR has the potential to provide an initial benchmark for the quality of surgery and anesthesia in a country or region. In LICs, the operating logbook is omnipresent, and therefore collecting the POMR 24 will be a simple matter and is unlikely to burden a system stretched thin in most LICs. Reporting the POMR will require support from Ministries of Health and the World Health Organization to insure appropriate comparison and analysis. 
The POMR is not intended for granular comparison of institutions or departments. As a health indicator, the POMR reveals death related to anesthesia and surgery generally. Collecting additional information on anesthesia and surgical complications, including the specific circumstances of death, would be far more valuable. For this reason the POMR 30, defined as death prior to discharge or within 30 days of surgery, including stratifies such as ASA (American Society of Anesthesiologists) status, age, surgical urgency (emergent or essential), procedure type, and suspected contributor (hemorrhage, infection, myocardial infarction, pulmonary embolus, etc.) would allow for meaningful analysis. In LICs following and evaluating patients preoperatively represents a significant challenge.

A representative, practical health indicator for surgery and safe anesthesia is critical as surgery in LICs increases in scope to treat a new era of NCDs (chronic non-communicable diseases). The POMR is an initial indicator that can provide valuable insight into the state of surgical care, and that may provide an initial benchmark for improvement. It is nonspecific and therefore flawed, but it has value as a concrete, initial indicator. A surgical health indicator is an essential next step for the inclusion of surgical services in global health and for prioritization within the Post-2015 health goals.

\section{References}

1. Lozano R, Naghavi M, Foreman K et al (2010) Global and regional mortality from 235 causes of death for 20 age groups in 1990 and 2010: a systematic analysis for the Global Burden of Disease Study. Lancet 380(9859):2095-2128

2. McQueen KA, Ozgediz D, Riviello R et al (2010) Essential surgery: integral to the right to health. Health Hum Rights J 12(1):137-152

3. Mock C, Cherian M, Juliard C et al (2010) Developing global priorities in addressing surgical conditions-furthering the link between surgery and public health. World J Surg 34:381-385. doi:10.1007/s00268-009-0263-4

4. Hansen D, Gausi SC, Merikebu M (2000) Anaesthesia in Malawi: complications and deaths. Trop Doct 30:146-149

5. Hodges SC, Mijumbi C, Okello M et al (2007) Anaesthesia services in developing countries: defining the problems. Anaesthesia 62:4-11

6. Khan MU, Khan FA (2006) Anaesthesia-related mortality in developing countries. Anaesth Intensive Care 34:523-524

7. Mathers CD, Fat DM, Idoue MF et al (2005) Counting the dead and what they died from: an assessment of the global status of cause of death data. Bull WHO 83:171-177

8. Royal College of Australasian Surgeons (2012) Discussions and follow up to the 6th Annual ASAP Meeting. Melbourne, Australia 